EPJ Web of Conferences 41, 05032 (2013)

DOI: $10.1051 /$ epjconf/20134105032

(C) Owned by the authors, published by EDP Sciences, 2013

\title{
Two-Dimensional Electronic Spectroscopy of a Model Dimer System
}

A. Halpin ${ }^{1}$, P.J.M. Johnson ${ }^{1}$, R.S. Murphy ${ }^{2}$, V.I. Prokhorenko ${ }^{3}$, and R.J.D. Miller ${ }^{1,3, a}$

1 Institute for Optical Sciences and Departments of Chemistry and Physics, University of Toronto, 80 St. George Street, Toronto, Ontario M5S 3H6, Canada.

2 Department of Chemistry and Biochemistry, University of Regina, 3737 Wascana Parkway, Regina, SK, S4S 0A2, Canada.

3 Max Planck Research Department for Structural Dynamics, Department of Physics, University of Hamburg, Centre for Free Electron Laser Science, DESY, Notkestrasse 85, D-22607 Hamburg, Germany.

\begin{abstract}
Two-dimensional spectra of a dimer were measured to determine the timescale for electronic decoherence at room temperature. Anti-correlated beats in the crosspeaks were observed only during the period corresponding to the measured homogeneous lifetime.
\end{abstract}

\section{Introduction}

Two-Dimensional Photon-Echo Spectroscopy (2DPE) has become a widely used tool in the study of molecular aggregates. By providing a second frequency dimension on which the optical response can be mapped, it is possible to investigate electronic couplings between tightly packed pigments in photosynthetic proteins as well as exciton transport in self assembling aggregates of organic dyes [1] . Great emphasis has been placed on measuring the dynamics of off-diagonal elements of the 2D spectra or cross peaks, as oscillations of these features can be the signature of electronic coherences between states. It has been argued that electronic decoherence should occur too rapidly at room temperature for electronic coherences between chromophores to affect the mechanism of energy transfer between pigment aggregates. However, contrary to this line of thought, wavelike energy transfer has been reported in a number of measurements on the proteins involved in photosynthesis [2]. In this work we investigate electronic coherences in the simplest molecular aggregate: a covalently bound homodimer. Two indocarbocyanine dyes are linked via two chains with four linkers, which act to "tune" the dipoledipole coupling responsible for splitting the monomer S1 state into two delocalized exciton states. This coupling is critically sensitive to both the distance and angle between dipoles, so linking the monomers rigidly can greatly restrict the possible conformations, mimicking the reality in proteins where each pigment is fixed in its binding pocket and relative orientations between pigments are well defined. Due to strong coupling between subunits, we can resolve cross peaks that are completely distinct and separated from their diagonal counterparts.

\section{Experimental Methods}

Measurements were performed using the output of a non collinear optical parametric amplifier (NOPA) as well as a broadband anamorphic pump NOPA [3] with all reflective seed optics to enhance phase

\footnotetext{
a e-mail: dwayne.miller@mpsd.cfel.de
} 


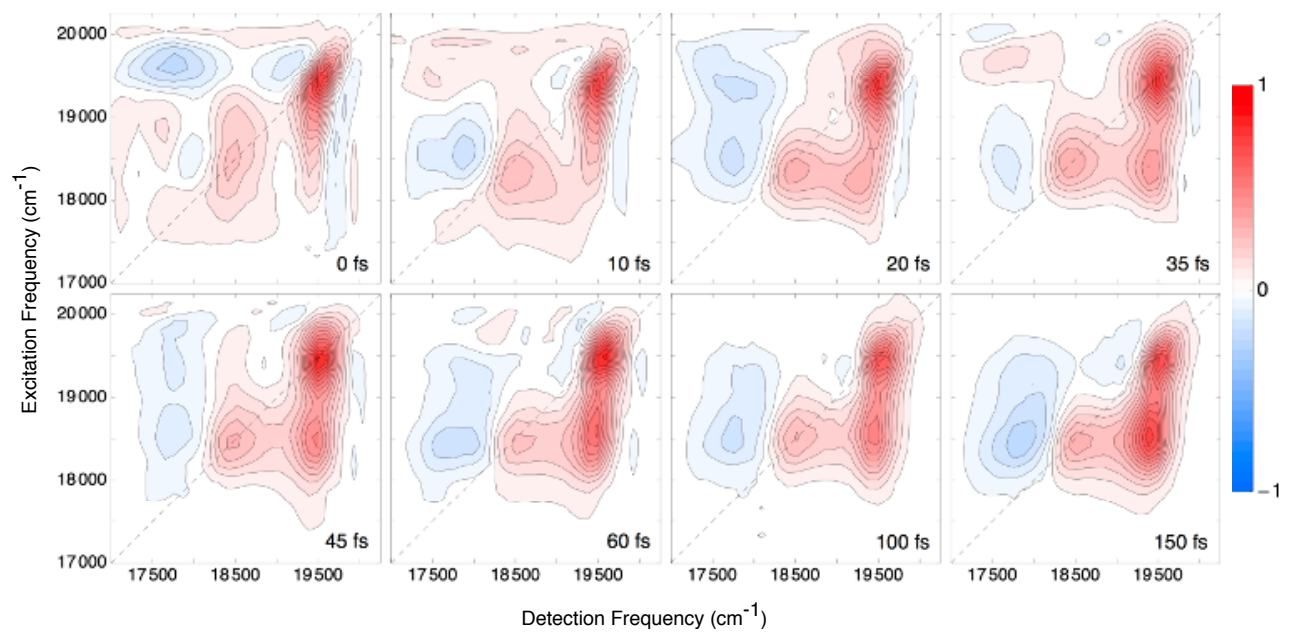

Fig. 1. Absorptive 2D Spectra of the biscyanine dimer at early waiting times ( $\leq 150 \mathrm{fs}$ ). Large amplitude oscillations in the off diagonal elements with a period of roughly $20 \mathrm{fs}$ appear during the first $50 \mathrm{fs}$, at which point the spectra become in large part static as electronic coherence has been lost.

matching in the blue part of the spectrum. We used p-polarized pulses of 16 fs and 12 fs duration respectively (measured in situ in a $1 \mathrm{~mm}$ thick fused silica window). The $2 \mathrm{D}$ spectrometer has been previously described in detail [4]. Pulse compression was accomplished using a combination of a prism compressor and deformable mirror based pulse shaper (Oko Technology). The sample (dissolved in methanol) was circulated in a home built $250 \mu \mathrm{m}$ path length flow cell, with an optical density of 0.25 (at $18400 \mathrm{~cm}^{-1}$ ), and $150 \mu \mathrm{m}$ thick fused silica windows to minimize non-resonant response in the area of pulse overlap. 2DPE spectra were collected for the first $150 \mathrm{fs}$ of waiting times at intervals of 5 fs. The heterodyne detected photon echo signals were measured by scanning the $\tau$ coordinate over the range of $[-128 ; 128]$ fs in steps of $1 \mathrm{fs}$.

\subsection{Results and Discussion}

The two major peaks in the absorption spectrum of the dimer (not shown), at $19700 \mathrm{~cm}^{-1}$ and 18400 $\mathrm{cm}^{-1}$, are immediately identifiable in the 2D spectra shown in Fig 1. The crosspeaks between these states are clearly resolved and separate from the diagonal peaks due to the large energy split in the system. We also measure strong excited state absorption on the red side of the spectra, which is also present in separate measurements on the monomer. In early reports on biscyanines these two peaks have been assigned to the two first-exciton states produced by Davydov splitting in the system. More recent semiclassical and ab-initio calculations have posited that the $18400 \mathrm{~cm}^{-1}$ secondary peak arises in fact due to vibronic coupling [5]. This work suggests that the redshifted shoulder we observe at $17400 \mathrm{~cm}^{-1}$ is due to the exciton with opposite electronic parity to the main band at $19700 \mathrm{~cm}^{-1}$. This hypothesis is corroborated by the $2 \mathrm{D}$ spectra, as we see very dramatic oscillations at the corresponding locations in the spectra. In Fig 1, the spectra for $\mathrm{T}=10 \mathrm{fs}$ and $\mathrm{T}=35 \mathrm{fs}$ show this effect plainly: despite the weak signal on the diagonal at $17400 \mathrm{~cm}^{-1}$ its coupling to the main band leads to a crosspeak of $\pm 15 \%$ of the maximum signal amplitude. We extracted the dynamics of the cross peaks below and above the diagonal, and found that they exhibit anti-correlated oscillations at early evolution times. Fitting each curve to a single decaying sinusoid (dashed/dotted lines, Fig 2) yielded identical oscillation periods and a decay rate corresponding to approximately $45 \mathrm{fs}$. In a separate measurement, we measured the homogeneous line width of the main band at $19700 \mathrm{~cm}^{-1}$ and recovered an electronic decoherence time corresponding to $40 \mathrm{fs}$. The sub diagonal signals have a strong contribution from the ground state bleach signal of the main band, but it is interesting to note that since the shoulder at $17400 \mathrm{~cm}^{-1}$ absorbs so weakly, that the oscillating cross peak associated to it above the diagonal 




Fig. 2. Dynamics of cross peak amplitudes between the $19700 \mathrm{~cm}^{-1}$ and $17400 \mathrm{~cm}^{-1}$ transitions showing clear anti correlation at early times. Fits to a single decaying sinusoid are shown in dashed/dotted lines for above/below diagonal cross peaks.

can be recorded "background free". It only appears during the coherent portion of the evolution, then vanishes to give way to the static ESA feature once electronic coherence is lost. A final interesting feature of this cross peak, is that it does not appear immediately at $\mathrm{T}=0 \mathrm{fs}$, but only achieves maximal amplitude $10 \mathrm{fs}$ following excitation. The interpretation of this effect is still under question.

\section{References}

1. Milota, F., Sperling, J., Nemeth, A., Mančal, T., \& Kauffmann, H. F. Accounts of Chemical Research 42, 1364-1374 (2009).

2. Collini, E., Wong, C. Y., Wilk, K. E., Curmi, P. M. G., Brumer, P., \& Scholes, G. D., Nature 463, 644-647 (2010).

3. Johnson, P. J. M., Prokhorenko, V. I., \& Miller, R. J. D., Optics letters 36, 2170-2172 (2011).

4. Prokhorenko, V. I., Halpin, A., \& Miller, R. J. D., Optics express 17, 9764-9779 (2009).

5. Eisfeld, A., Braun, L., Strunz, W. T., Briggs, J. S., Beck, J., \& Engel, V., Journal of Chemical Physics 122, 134103 (2005). 